\title{
Kirchliche Filmpreise 2004
}

Aufgeführt ist im folgenden eine Auswahl der Preise von Jurys der kirchlichen Filmorganisationen SIGNIS (katholisch) und INTERFILM (evangelisch), die im Jahre 2004 auf wichtigen internationalen Filmfestivals vergeben wurden.

\section{Internationale Filmfestspiele Berlin [5.2. bis 15.2.2004)}

Die Ökumenische Jury vergibt in Berlin Preise in den Programmsektionen Internationaler Wettbewerb, Internationales Forum des Jungen Films und Panorama.

Sektion Wettbewerb

Preis:

Just a Kiss (OT: A Fond Kiss)

Großbritannien/Italien/Deutschland/Spanien 2003

Regie: Ken Loach

Begründung:

Am Beispiel der Liebesgeschichte zwischen einem jungen Mann pakistanischer Herkunft und einer irischen Lehrerin thematisiert der Film die kulturellen, religiösen und gesellschaftlichen Fronten, die es für ein gemeinsames Zusammenleben zu überwinden gilt. Ein überzeugendes Plädoyer für Toleranz und interkulturelle Verständigung.

Lobende Erwähnung:

Svjedoci (Die Zeugen)

Kroatien 2003

Regie: Vinko Brešan

Begründung:

Auf ausgezeichnete Weise setzt der Film künstlerische Mittel ein, um die moralischen Probleme in einer Kriegssituation darzustellen. Er zeigt, dass sogar in dieser Situation humanes Handeln möglich ist. Der einzige Weg zu einer friedlichen Zukunft gelingt nur dort, wo das Risiko übernommen wird, Leben zu schützen und sogar der Respekt gegenüber den Feinden als menschlichen Wesen gewahrt bleibt. 
Sektion Panorama

Preis

Mi piace lavorare (Mobbing) (Ich liebe meine Arbeit [Mobbing])

Italien 2003

Regie: Francesca Comencini

Begründung:

Der Film beschreibt eindrücklich das Leiden einer allein erziehenden Frau, die an ihrem Arbeitsplatz ungerecht behandelt wird und dem Mobbing von Mitarbeitern ausgesetzt ist. Die Regisseurin betont auf herausfordernde und angemessene Weise das Recht auf Gerechtigkeit und menschliche Würde.

Sektion Internationales Forum des Jungen Films

Preis

Folle embellie (Schöne Phantasie)

Frankreich/Belgien/Kanada 2003

Regie: Dominique Cabrera

Begründung:

„Folle embellie“ ist zugleich eine einfühlsame Geschichte und eine umfassende Metapher. Der Film setzt den Wahnsinn des Krieges gegen die Krankheit von geistig Behinderten und fragt nach den Möglichkeiten der Heilung durch gemeinsames Handeln und gelingende menschliche Beziehungen.

\section{Internationale Kurzfilmtage Oberhausen (29.4. bis}

\subsubsection{4)}

Preis der Ökumenischen Jury

Britanya

Niederlande 2003

Regie: Marjoleine Bonnstra

Begründung:

Dieses Video ragt aus den zahlreichen Filmen zum Thema Migration deutlich heraus. Zurückhaltend und sensibel gibt es Menschen, die nur ihre Hoffnung haben, Gesichter und Würde, Raum und Zeit. Bemerkenswert sind seine herausfordernde Filmsprache, seine ruhigen Einstellungen und seine beeindruckende Symbolik. 
Lobende Erwähnungen

Les Tartines (Schnittchen)

Schweiz 2003

Regie: Anthony Vouardoux und Tania Zambrano-Ovalle

Begründung:

Frisch, leicht und originell, humorvoll und hintergründig spielt „Les Tartines“ mit der Bedeutung von Liebe, Sexualität, Essen und Sprache.

\section{WASP}

Großbritannien 2003

Regie: Andrea Arnold

Begrïndung:

Armut, Muttersein, Hoffnung auf ein besseres Leben - darum geht es in „WASP“. Humorvoll und spannend zugleich gestaltet der Film diese brisanten Themen. Im Dogma-Stil gedreht, lebt „WASP“ von seinen hervorragenden schauspielerischen Leistungen, dem fesselnden Soundtrack und einer überzeugenden Dramaturgie.

\section{Internationale Filmfestspiele Cannes (12.5. bis 23.5.2004)}

Preis der Ökumenischen Jury

Die Reise des jungen Che - Motorcycle Diaries (OT: Diarios de motocicleta) USA/Deutschland/Argentinien/Großbritannien 2004

Regie: Walter Salles

Begründung:

Ausgehend von den Reisetagebüchern Ernesto Che Guevaras, schildert Walter Salles den Bewusstwerdungsprozess des jungen Che. Mit den Stilmitteln des Roadmovies zeigt er, wie der junge Medizinstudent sein Mitgefühl für die Anderen in ihrem Leiden entdeckt. Hier entsteht sein Bewusstsein für die lateinamerikanische Realität seiner Zeit. Wir sehen, wie Ches Verlangen nach sozialer Gerechtigkeit wächst. Der Film lenkt die Aufmerksamkeit auf einen Kontintent, dessen soziale und politische Probleme bis heute auf eine Lösung warten.

Lobende Erwähnung:

\section{Moolaadé}

Senegal 2004

Regie: Ousmane Sembène

„Moolaadé“ kritisiert einen extremen Islamismus, der die Religion missbraucht, um junge Mädchen zur Klitorisbeschneidung zu zwingen. In Ousmane Sembènes Film fordern die Frauen eines senegalesischen Dor- 
fes die Abschaffung dieses Rituals. Der Film setzt das ländliche Alltagsleben präzise und farbenreich in Szene. Er erzählt eine Geschichte, in der Tradition und Moderne miteinander verwoben sind und in der das Dorf zur Theaterbühne eines Lehrstücks wird.

\section{Internationales Filmfestival Locarno (4.8. bis 14.8.2004)}

Preis der Ökumenischen Jury:

Yasmin

Großbritannien, Deutschland 2004

Regie: Kenny Glenaan

Begründung:

Durch die Folgen des 11. Septembers aufgerüttelt, findet Yasmin zu ihren kulturellen Wurzeln zurück. Die Wiederentdeckung des Korans bringt eine Glaubensvertiefung mit sich und ermöglicht ihr, sich sebst $z \mathfrak{u}$ finden. Zuversichtlich sucht Yasmin einen Weg, sich zu behaupten bis sowohl in der pakistanischen Gemeinschaft wie auch in ihrer westlich geprägten Umgebung.

Lobende Erwähnung:

Private

Italien 2004

Regie: Saverio Castanzo

"Private“ zeigt uns das Leben einer palästinensischen Familie, die sich weigert, ihr von israelischen Truppen besetztes Haus zu verlassen. Ihre Weigerung gründet in einer überzeugten, wenngleich paternalistischen Haltung der Gewaltlosigkeit. Das Leben in dieser "geschlossenen Gesellschaft" beleuchtet die verschiedenen Verhaltensweisen im Alltag der kriegerischen Auseinandersetzung. Der Film setzt ein Zeichen der Hoffnung für unsere heutige Welt.

\section{Internationales Filmfestival Venedig (1.9. bis 11.9.2004)}

Preis der internationalen katholischen Filmorganisation SIGNIS

Tout un hiver sans feu (Ein ganzer Winter ohne Feuer)

Schweiz/Belgien 2004

Regie: Greg Zglinski

Begründung:

Das Thema des geteilten Leids zwischen den Schweizern und den Kosovo-Flüchtlingen wird in einem sehr strengen und disziplinierten Stil 
dargeboten. Der Film zeigt die Möglichkeit des Neubeginns durch Rückbesinnung auf tiefere, authentischere Werte.

Lobende Erwähnung:

Binjip

Südkorea 2004

Regie: Kim Ki-duk

Begründung:

Eine symbolische Geschichte über einen jungen Mann, der leere Häuser besetzt. Das Thema der Überwindung von Grenzen der physischen Welt, damit Menschen zu spirituellen Dimensionen vordringen können, wird gleichermaßen feinfühlig wie schön behandelt.

\section{Internationales Leipziger Festival für Dokumentar- und Animationsfilm (19.10. bis 24.10.2004)}

Der Preis der Ökumenischen Jury

Am seidenen Faden

Deutschland 2004

Regie: Katarina Peters

Begründung:

In ihrem Filmtagebuch dokumentiert die Regisseurin den Heilungsprozess ihres Mannes (eines jungen Musikers) nach einem schweren Gehirnschlaganfall. Die Erzählweise gewährt uns einen sehr intimen Einblick in das nachhaltig veränderte Leben beider, in den Kampf um ihre Ehe und ihre zukünftige Existenz. Zweifel und Gefährdungen verschweigt dieser berührende Film über die Bewältigung von Schicksal „am seidenen Faden" nicht: ein Exempel für Liebe und Verantwortungsbereitschaft, für Mut und Lebenswillen!

\section{Festival des osteuropäischen Films in Cottbus (2.11. bis 6.11.2004)}

Preis der Ökumenischen Jury

Kontroll (Kontrolle)

Ungarn 2003

Regie: Nimród Antal

Begründung:

Die Budapester Metro wird zu einem packenden Gleichnis für die verborgenen Seiten der menschlichen Existenz - sowohl in ihrer sozialen wie in 
ihrer psychischen Dimension. Mit großer Genauigkeit verknüpft der Regisseur die Abgründe unserer Gesellschaft mit den Abgründen der menschlichen Seele. Er lotet ihre Gefahren in aller Schärfe aus, ohne die Hoffnung auf Rettung aus dem Auge zu verlieren.

\section{Internationales Filmfestival Mannheim-Heidelberg (18.11. bis 27.11.2004)}

Preis der Ökumenischen Jury

Mila ot Mars (Mila vom Mars)

Bulgarien 2004 Regie: Zornitsa Sophia

Begründung:

Der Film begleitet eine junge Frau, die auf einem mühsamen Weg in der Begegnung mit Menschen an einem abgeschiedenen Ort zu ihrer Identität findet. Das vielschichtige Erstlingswerk spielt mit Motiven aus der christlichen Tradition und überzeugt durch Bildkomposition, Montage und schauspielerische Leistung.

Lobende Erwähnung

Nema Problema (Keine Probleme)

Italien 2004

Regie: Giancarlo Bocchi

Begründung:

Der Film schildert in stringenten Bildern die schwierige Situation journalistischer Arbeit im Krieg. Er thematisiert Mechanismen wechselseitiger Manipulation und ruft ins Bewusstsein, dass die Wahrheitssuche bedingungslose Sorge der Demokratie bleiben muss.

Zusammengestellt von Peter Hasenberg 